\title{
ARTICLE \\ Overexpression of corticotropin-releasing factor in the nucleus accumbens enhances the reinforcing effects of nicotine in intact female versus male and ovariectomized female rats
}

\author{
Kevin P. Uribe ${ }^{1}$, Victor L. Correa ${ }^{1}$, Briana E. Pinales ${ }^{2}$, Rodolfo J. Flores (D) ${ }^{1}$, Bryan Cruz (D) $^{1}$, Zhiying Shan ${ }^{3}$, Adriaan W. Bruijnzeel ${ }^{4}$, \\ Arshad M. Khan $\mathbb{i D}^{2,5}$ and Laura E. O'Dell ${ }^{1,5}$
}

\begin{abstract}
This study assessed the role of stress systems in the nucleus accumbens (NAc) in promoting sex differences in the reinforcing effects of nicotine. Intravenous self-administration (IVSA) of various doses of nicotine was compared following overexpression of corticotropin-releasing factor (CRF) in the NAc of female and male rats. Ovariectomized (OVX) females were also included to assess the role of ovarian hormones in promoting nicotine reinforcement. Rats received intra-NAc administration of an adeno-associated vector that overexpressed CRF (AAV2/5-CRF) or green fluorescent protein (AAV2/5-GFP). All rats were then given extended access $(23 \mathrm{~h} /$ day) to an inactive and an active lever that delivered nicotine. Separate groups of rats received intra-NAc AAV2/5-CRF and saline IVSA. Rats were also allowed to nose-poke for food and water during IVSA testing. At the end of the study, the NAc was dissected and rt-qPCR methods were used to estimate CRF overexpression and changes in CRF receptors (CRFr1, CRFr2) and the CRF receptor internalizing protein, $\beta$-arrestin2 (Arrb2). Overexpression of CRF in the NAc increased nicotine IVSA to a larger extent in intact female versus male and OVX females. Food intake was increased to a larger extent in intact and OVX females as compared to males. The increase in CRF gene expression was similar across all groups; however, in females, overexpression of CRF resulted in a larger increase in CRFr1 and CRFr2 relative to males. In males, overexpression of CRF produced a larger increase in Arrb2 than females, suggesting greater CRF receptor internalization. Our results suggest that stress systems in the NAc promote the reinforcing effectiveness of nicotine in female rats in an ovarian hormone-dependent manner.
\end{abstract}

Neuropsychopharmacology (2020) 45:394-403; https://doi.org/10.1038/s41386-019-0543-0

\section{INTRODUCTION}

Tobacco use, primarily driven by nicotine, remains the leading cause of preventable deaths in the United States [1]. Clinical studies have revealed that the degree to which stress promotes tobacco use is greater in women versus men. Specifically, women are more likely to smoke to alleviate negative affective states and regulate mood as compared to men [2-4]. There is also a stronger comorbid association between stress and smoking in females, as women with a prior history of an anxiety disorder display higher smoking relapse rates as compared to men [5, 6]. Additionally, women with post-traumatic stress disorder report higher ratings of negative affect during smoking abstinence as compared to men [7]. Women also report a greater expectancy that smoking will relieve negative affective states during abstinence than men [8]. Stress has also been shown to enhance self-reports of nicotine craving and increase the likelihood of smoking relapse to a larger extent in women versus men [9]. In order to reduce health disparities produced by tobacco use in women, there is a need to understand the mechanisms by which stress promotes nicotine use in females.

The hypothalamic-pituitary-adrenal (HPA) axis coordinates the stress response via the release of the neuropeptide, corticotropin- releasing factor (CRF). Changes in CRF systems can serve as a neurobiological marker of chronic changes in stress responsiveness. Previous reports in rodents have revealed that extinguished responding for nicotine is reinstated following administration of the pharmacological stressor, yohimbine [10]. Nicotine-seeking behavior is also reinstated following restraint stress and footshock in rats $[11,12]$. These effects seem to be mediated via the CRF system as administration of the nonspecific CRF receptor antagonist, D-Phe CRF, decreases stress-induced reinstatement of nicotine-seeking behavior [13]. In addition, stress-induced elevations in active lever responding are prevented by administration of a CRFr1 receptor antagonist in male rats [14]. These studies suggest that CRF systems play a role in promoting nicotine-seeking behavior during extinction.

There are CRF receptors (CRFr1 and CRFr2) throughout the mesolimbic pathway $[15,16]$, which originates in the ventral tegmental area (VTA) and terminates in several forebrain structures, including the nucleus accumbens (NAc). The presence of CRF receptors in the mesolimbic pathway provides an anatomical substrate by which stress systems can promote the reinforcing effects of nicotine. For example, a previous study revealed that CRFr1 and CRFr2 are localized on dopaminergic

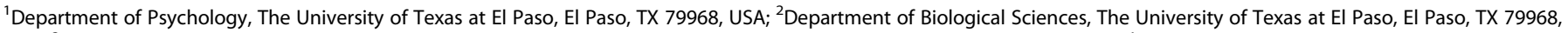

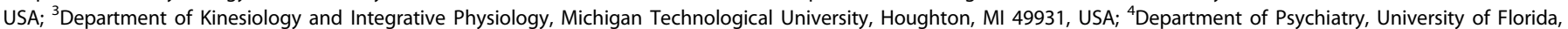
Gainesville, FL 32611, USA and ${ }^{5}$ Border Biomedical Research Center, The University of Texas at El Paso, El Paso, TX 79968, USA Correspondence: Laura E. O'Dell (lodell@utep.edu)

Received: 15 May 2019 Revised: 1 October 2019 Accepted: 7 October 2019 Published online: 15 October 2019 


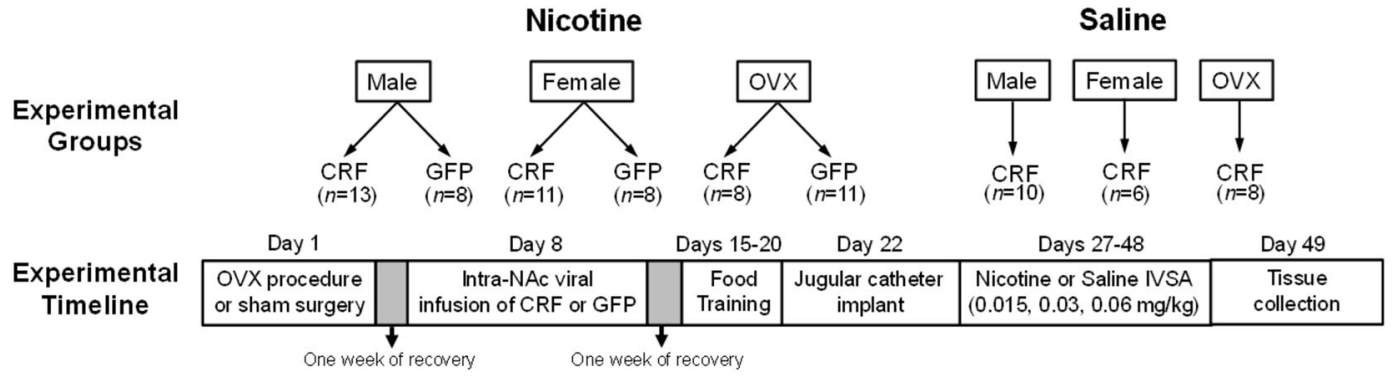

Fig. 1 This diagram reflects the experimental treatment groups and overall timeline

terminals in the NAc [17], suggesting that CRF regulates dopamine release in the NAc. This same report also found that intra-NAc infusions of CRF produce conditioned place preference (CPP) in mice, an effect that was ameliorated following lesions of dopamine neurons in the NAc. To our knowledge, a role for CRF systems in the NAc in promoting the reinforcing effects of nicotine has not yet been reported. Thus, this study assessed whether CRF systems in the NAc promote the reinforcing effects of nicotine in a sex- and ovarian hormone-dependent manner.

\section{MATERIALS AND METHODS}

Subjects

The rats for this study were bred from an outbred stock of female and male Wistar rats (Envigo, Inc.) were pair-housed with a samesex litter mate on a $12 \mathrm{~h}$ light/dark cycle (lights on at 6 a.m.) with ad libitum access to food and water. All procedures were approved by the UTEP Institutional Animal Care and Use Committee. Figure 1 depicts the overall experimental timeline. Briefly, rats received either intra-NAc infusions of AAV2/5-CRF or AAV2/5-GFP (green fluorescent protein). After 3 weeks, rats received extended ( $23 \mathrm{~h} /$ day) access to saline or nicotine IVSA in 4-day cycles with 3 days of forced abstinence. In a separate study, rats received intra-NAc infusions of AAV2/5-GFP, and 3 weeks later, the rats were sacrificed and the brains were collected for GFP immunohistochemistry.

\section{Surgical procedures}

At post-natal day (PND) 45, a group of female rats were anesthetized with an isoflurane/oxygen vapor mixture (1-3\%) and received either an ovariectomy or a sham surgical procedure. An incision was made about $1 \mathrm{~cm}$ medial to the knee and $2 \mathrm{~cm}$ lateral to the spinal cord where the ovaries were retracted and removed. Male rats also received a sham surgical procedure at PND 45. For the sham procedure, an incision was made in the same location as the ovariectomy procedure. A week later, the rats were re-anesthetized and received a bilateral infusion of a viral vector that overexpresses CRF (AAV2/5-CRF) or GFP (AAV2/5GFP). The infusions were aimed at the shell of the NAc using the following coordinates from Bregma (in $\mathrm{mm}$ : $\mathrm{AP}=+1.7 ; \mathrm{ML}=$ \pm 1.4 ; $D V=-8.1$ ) [18]. The viral vectors were obtained from a commercial vendor (Vector BioLabs). Previous work revealed that this viral construct (AAV2/5) increased GFP expression 2 weeks after intra-amygdala infusions, and the level of viral overexpression was maintained for 8 weeks [19]. The same report revealed that this viral construct increased CRF mRNA and protein levels in the amygdala relative to GFP controls. Separate groups of rats received bilateral infusions of AAV2/5-CRF or AAV2/5-GFP using an injection volume of $0.5 \mu \mathrm{l}$ over a 3 -min period. Following the infusion, the injectors were left in place for an additional $5 \mathrm{~min}$ to allow proper diffusion from the injector tip. A week later, the rats were prepared with IV catheters [20]. Briefly, silastic tubing was inserted into the jugular vein and anchored to surrounding tissue. The free end of the catheter contained an exit port that was attached to an acrylic-based pedestal that was anchored on the rats back. Catheters were flushed every other day with cefazolin antibiotic $(50 \mathrm{mg} / \mathrm{mL}$ ) and heparinized saline (30 USP units $/ \mathrm{mL}$ ).

\section{IVSA methods}

Operant chambers (Med associates, Inc.) were kept on the same $12 \mathrm{~h}$ light/dark cycle as the holding room. Prior to IVSA testing, the rats were trained using extended ( $23 \mathrm{~h} /$ day) access procedures to perform operant responses for food and water for 5 days on a fixed-ratio (FR) 1 schedule of reinforcement. The rats were allowed to nose-poke in a food receptacle that delivered palatable chow from a pellet dispenser $(45 \mathrm{mg} / \mathrm{kg}$; BioServ Inc.). The rats also performed nose-poke responses in a separate hole for the delivery of $0.1 \mathrm{~mL}$ of water into an adjacent dipper cup. All rats reached stable levels of responding after 5 days of food and water training. Following catherization surgery, the rats were allowed to recover in their home cage for 5 days. In the first IVSA session, two novel levers were introduced (active and inactive). Responses on the active lever resulted in a 1-s infusion of saline or nicotine (FR1), and responses on the inactive lever had no scheduled consequences. The onset of the infusion was signaled by a cue light above the active lever that was illuminated for a 20-s timeout period. (-) Nicotine hydrogen ditartrate (NIDA, Research Triangle Institute) was dissolved in $0.9 \%$ sterile saline ( $\mathrm{pH}$ of 7.4 ), and different concentrations of nicotine were prepared daily based on the weight of the rat from the previous day. An escalating dose regimen was employed, whereby rats received saline or increasing doses of nicotine in the following order, 0.015 then 0.03 and then $0.06 \mathrm{mg} / \mathrm{kg} / 0.1 \mathrm{~mL}$ per infusion (expressed as base). The rats were given access to each dose in separate 4-day cycles with 3 intervening days of nicotine abstinence prior to initiating the next dose of nicotine. Food and water intake were continuously recorded throughout IVSA testing. Operant responses for food were normalized as per Kleiber's law as a power function of the animals' daily body weight to account for increased metabolic needs and body mass across time [21, 22].

\section{Gene expression methods}

The day after the last IVSA session, the rats were decapitated and the NAc was dissected from the brain, flash-frozen, and stored in the $-80^{\circ} \mathrm{C}$ freezer. Total RNA was isolated using the RNeasy mini kit (Qiagen, Inc.). Real-time quantitative polymerase chain reaction (rt-qPCR) methods were used to assess gene expression of CRF, CRFr1, CRFr2, and $\beta$-arrestin2 (Arrb2). PCR values were normalized to RPL13a based on previous work showing that this housekeeping gene is most stable and similar in female and male rats [23]. The assessment of gene expression was done in a tissue punch of the NAc. Although every attempt was made to isolate the tissue punch to the shell region, there may have been portions of the core that may have been included. To address this issue, rats were excluded that did not display similar gene expression levels as their group. This elimination criterion was employed for the housekeeping gene as well as CRF. The CDNA primer sequences are illustrated below. 
396

$\begin{array}{lll}\text { Gene } & \begin{array}{l}\text { Forward primer sequence } \\ \left(5^{\prime} \text { to } 3^{\prime}\right)\end{array} & \begin{array}{l}\text { Reverse primer sequence } \\ \left(3^{\prime} \text { to } 5^{\prime}\right)\end{array}\end{array}$

RPL13a GGA TCC CTC CAC CCT ATG ACA CTG GTA CTT CCA CCC GAC CTC CRF ATG CTG CTG GTG GCT CTG T GGA TCA GAA TCG GCT GAG GT

CRFr1 CCA GAG CAA TGT GGC

CRFr2 TCA TTG GAT GGT GCA TAC CTG TGT GCA GGT AGC

Arrb2 CTG AAA CCA CAC GCC TTG ATG AGG AGC ACG AG GTG ACG TGG ACG TTG

Immunohistochemistry procedures

A separate cohort of female and male rats $(n=3)$ received viral overexpression of GFP in the NAc, as described above. Three weeks later, the rats were prepared for immunohistochemistry as described previously [24]. Briefly, subjects were anesthetized and transcardially perfused with $100 \mathrm{~mL}$ of $0.01 \mathrm{M}$ sodium phosphatebuffered saline ( $\mathrm{pH} 7.4$ at room temp) and then with $300 \mathrm{~mL}$ of $4 \%$ paraformaldehyde solution ( $\mathrm{pH} 9.5$ at $4{ }^{\circ} \mathrm{C}$ ). Frozen blocks of fixed brain tissue were then cut into $30-\mu \mathrm{m}$-thick coronal sections and collected into 24-well trays filled with cryoprotectant. Individual brain sections containing the NAc and the VTA were incubated at $4{ }^{\circ} \mathrm{C}$ for $15-21 \mathrm{~h}$ with an anti-GFP chicken IgY antibody solution (1:1000 dilution; Aves Labs, Inc) and an anti-neuronal nuclei (NeuN) mouse monoclonal lgG (1:8000 dilution; EMD-Millipore, Inc). The sections were then rinsed and incubated at room temperature with a cocktail of secondary antibodies (donkey antichicken IgY biotin and donkey anti-mouse IgG Cy3; Jackson ImmunoReseach, Inc.; 1:250 final dilutions for each) in blocking solution. After incubation, tissue sections were rinsed and incubated with streptavidin Alexa Fluor 488 conjugate $(1: 1000)$ and diamindino-2-phenylindole dihydrochloride (DAPI; 1:4000; ThermoFisher Scientific, Inc) for $1 \mathrm{~h}$. Sections were mounted and coverslipped using sodium bicarbonate-buffered glycerol $(\mathrm{pH} 8.6$ at room temp) and then stored at $4{ }^{\circ} \mathrm{C}$. To visualize neuronal cytoarchitecture, sections from an adjacent tissue series were processed for Nissl staining. Sections were mounted, dehydrated with ascending ethanol, defatted in xylene, stained using $0.5 \%$ thionin acetate (Sigma-Aldrich, Inc), differentiated in $0.4 \%$ glacial acetic acid, and then coverslipped with mounting medium DPX (VWR, Inc.). To visualize the spread of GFP, the tissue was examined with a Zeiss M2 Axioimager using filter sets appropriate for the fluorophores used. The microscope was connected to a cooled EXi Blue CCD camera (QImaging) driven by Volocity Software (Perkin-Elmer, Inc).

Statistical analyses

All behavioral data were analyzed using mixed-measures analysis of variance (ANOVA). The first level of analysis involved three separate ANOVAs in male, female, and OVX female rats with treatment group (Nicotine-CRF, Nicotine-GFP, and Saline-CRF) as a between-subject factor and days (1-12) as a within-subject factor. Where interaction effects were observed, the graphs illustrate the results of post-hoc comparisons between rats that received intraNAc GFP versus CRF following nicotine IVSA. The second level of analysis collapsed the data across all days of IVSA, and a one-way ANOVA was conducted across all groups. Where significant overall effects were observed, post-hoc analyses were used to compare across groups using Fisher's LSD test $(p \leq 0.05)$. Tests for the assumption of multiple sphericity were conducted and violations were corrected using the Huynh-Feldt correction factor. This resulted in appropriate corrections to the degrees of freedom that resulted in more conservative $F$-ratios for the behavioral measures. For the rt-qPCR data, the delta-delta $\mathrm{Ct}$ method was used to calculate fold change for each gene [25]. The data were then analyzed using separate ANOVAs for each gene with group (male, intact female, and OVX female) and virus condition (GFP and CRF) as between-subject factors. The final analyses included rats that displayed behavioral or CRF gene expression values that fell within two standard deviations of the group mean. By this criterion six rats were removed from the following groups: two females from the Nicotine-CRF group, one male from the Nicotine-CRF group, one male from the Nicotine-GFP group, one OVX female from the Nicotine-GFP group, and one OVX female from the SalineCRF group.

To quantitatively assess the degree of colocalization of GFP and neuronal markers in the NAc, tissue was examined by a Zeiss LSM 700 confocal system (Carl Zeiss, Inc.). Regions of interest (ROIs) were delimited within cells to decrease the background fluorescence contribution. Using ZEN 2009 software (Carl Zeiss), colocalization was calculated using a squared Manders' overlap coefficient of the defined signals, performed on a pixel-by-pixel basis, which represented an accurate degree of colocalization. The overlap coefficient according to Manders' provided values within a range from 0 to 1 , with a value of 0 meaning that there were no pixels within the selected ROI with overlapped signals and a value of 1 representing perfectly colocalized pixels [26].

\section{RESULTS}

AAV vector transduces neurons in the NAC

Figure 2 depicts colocalization of GFP and the neuronal markers, NeuN and DAPI, in the shell of the NAc. An analysis of AAV2/5mediated transduction efficiency in neurons revealed that a majority of GFP-positive cells were DAPI- and NeuN-positive (90\%), suggesting that the cells that were transduced by the virus were neurons. Also, it does not appear that the virus was translocated to the VTA, as fluorescence was not detected in coronal slices of this region (data not shown).

\section{Overexpression of CRF in the NAc increases nicotine intake in intact females}

Figure 3 depicts daily nicotine intake in the top panels (Fig. 3a-c) and mean nicotine intake in the bottom panel (Fig. 3d). The analysis of daily nicotine intake revealed a two-way interaction between treatment group and days in male $\left(F_{(15.55,186.64)}=12.00\right.$, $p=0.001)$, intact female $\left(F_{(15.98,207.76)}=5.47, p=0.001\right)$, and OVX female $\left(F_{(14.23,170.74)}=11.41, p=0.001\right)$ rats. Post-hoc analyses revealed Nicotine-CRF intact females displayed greater nicotine intake on days $3-5,7,10$, and 12 as compared to Nicotine-GFP intact females $\left({ }^{*} p \leq 0.05\right)$. Also, Nicotine-CRF males and OVX females displayed greater nicotine intake on day 9 as compared to their respective GFP controls $\left({ }^{*} p \leq 0.05\right)$.

The analysis of mean nicotine intake revealed an overall difference across treatment groups $\left(F_{(8,74)}=83.06, p=0.001\right)$. Post-hoc analyses revealed Nicotine-CRF intact females displayed higher nicotine intake as compared to Nicotine-GFP intact females $\left({ }^{*} p \leq 0.05\right)$. Importantly, Nicotine-CRF intact females displayed higher nicotine intake as compared to Nicotine-CRF males ( ${ }^{\#} p \leq$ $0.05)$, and OVX females ( $\left.{ }^{\dagger} p \leq 0.05\right)$. In addition, Nicotine-GFP intact females displayed more nicotine intake as compared to NicotineGFP OVX females $\left({ }^{\dagger} p \leq 0.05\right)$. All Nicotine-CRF groups displayed greater intake as compared to their respective Saline-CRF groups $\left({ }^{@} p \leq 0.05\right)$.

Overexpression of CRF in the NAc increases active but not inactive responses in females

Figure 4 depicts active responses in the left panels (Fig. 4a-d) and inactive responses in the right panels (Fig. $4 \mathrm{e}-\mathrm{h}$ ). Daily measures are shown in the top panels (Fig. $4 \mathrm{a}-\mathrm{c}, \mathrm{e}-\mathrm{g}$ ) and mean responses in the bottom panels (Fig. $4 d, h$ ). The analyses of daily active responses revealed that there was no interaction between the treatment group and days in either intact females $\left(F_{(10.16,132.03)}=0.83, p=0.60\right)$ or OVX females $\left(F_{(9.56,109.95)}=0.67, p=0.74\right)$. However, there was a main effect of treatment group in intact females $\left(F_{(2,26)}=26.81\right.$, 
(A)

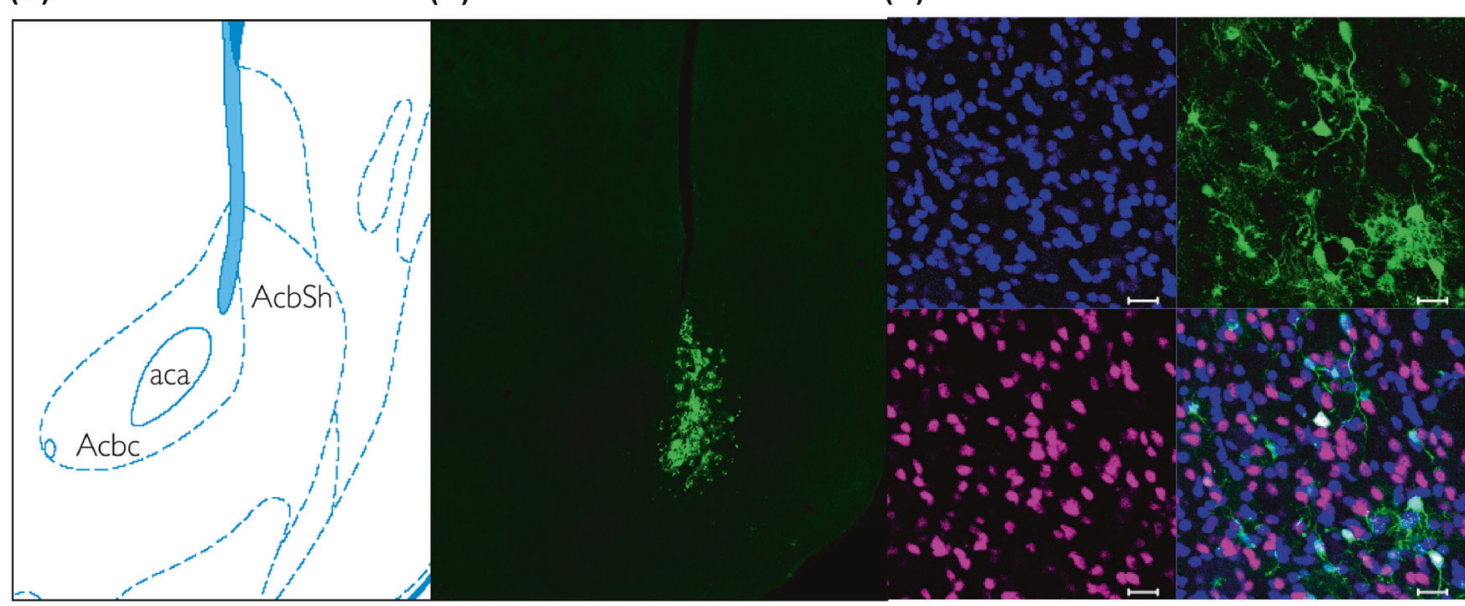

Fig. 2 AVV2/5-mediated expression of GFP in the NAc. The schematic (a) was adapted from the Paxinos and Watson atlas (2013) at AP $=+1.7 \mathrm{~mm}$ from Bregma to illustrate the NAc subdivisions of the core and shell. The confocal image from a representative female rat (b) illustrates that the spread of the AAV2/5-GFP vector was confined primarily to the NAc shell. Triple staining (c) for DAPI (blue), GFP (green), and NeuN (magenta) illustrates that $90 \%$ of GFP-expressing cells also express the neuronal markers, illustrating that the AAV2/5-transduced cells in the NAc are neurons. The scale bar is $20 \mu \mathrm{m}$

$p=0.001)$ and OVX females $\left(F_{(2,23)}=6.42, p=0.006\right)$. There was a significant two-way interaction between the treatment group and days in males $\left(F_{(15.32,183.81)}=1.99, p=0.018\right)$, with Nicotine-CRF males eliciting more active responses than Nicotine-GFP males on day $9\left(^{*} p \leq 0.05\right)$.

The analysis of mean active responses revealed an overall difference across treatment groups $\left(F_{(8,73)}=21.05, p=0.001\right)$. Post-hoc analyses revealed Nicotine-CRF intact female rats displayed more active responses versus Nicotine-GFP intact females $\left({ }^{*} p \leq 0.05\right)$. Importantly, Nicotine-CRF intact females displayed more active responses relative to Nicotine-CRF males $\left({ }^{\#} p \leq 0.05\right)$ and OVX females $\left({ }^{\dagger} p \leq 0.05\right)$. In addition, Nicotine-GFP intact females displayed greater mean active responses as compared to Nicotine-GFP males $\left({ }^{\#} p \leq 0.05\right)$ and OVX females $\left({ }^{\dagger} p \leq 0.05\right)$. All Nicotine-CRF groups displayed more nicotine intake as compared to their respective Saline-CRF groups ( $\left({ }^{@} p \leq 0.05\right.$ ).

The analyses of daily inactive responses revealed that there was no interaction between treatment group and days in either males $\left(F_{(10.65,127.81)}=1.59, p=0.11\right)$ or intact females $\left(F_{(11.83,153.79)}=1.60\right.$, $p=0.09)$. Also, there was no main effect of the treatment group in either males $\left(F_{(2,24)}=1.35, p=0.28\right)$ or intact females $\left(F_{(2,26)}=1.81, p=0.18\right)$. There was a significant two-way interaction between the treatment group and days in OVX females $\left(F_{(12.02,144.24)}=2.82, p=0.002\right)$, with Nicotine-CRF OVX females eliciting more inactive responses than Nicotine-GFP OVX females on day $3\left({ }^{*} p \leq 0.05\right)$.

The analysis of mean inactive responses revealed an overall difference across treatment groups $\left(F_{(8,74)}=3.17, p=0.01\right)$. Posthoc analyses revealed intact females displayed more inactive responses as compared to males, regardless of treatment group $(\# p \leq 0.05)$.

Overexpression of CRF in the NAc increases food intake in female rats

Figure 5 depicts daily food intake on the top panels (Fig. $5 a-c$ ) and mean food intake in the bottom panel (Fig. $5 \mathrm{~d}$ ). The analyses of daily food intake revealed that there was no interaction between the treatment group and days in intact females $\left(F_{(21.24,276.18)}=\right.$ $1.56, p=0.057)$. However, there was a main effect treatment group in intact female $\left(F_{(2,26)}=9.27, p=0.001\right)$ rats. There was a two-way interaction between treatment group and days in males $\left(F_{(16.12,193.48)}=1.80, p=0.033\right)$ and OVX females $\left(F_{(19.67,236.04)}=\right.$ $2.01, p=0.008)$. Post-hoc analyses revealed Nicotine-CRF OVX females displayed greater food intake on days $1,4-6$, and 10 as compared to their respective Nicotine-GFP group $\left({ }^{*} p \leq 0.05\right)$.

The analysis of mean food intake revealed an overall difference across treatment groups $\left(F_{(8,74)}=13.17, p=0.001\right)$. Post-hoc analyses revealed that Nicotine-CRF intact and OVX female rats elicited more food intake relative to their respective Nicotine-GFP group $\left({ }^{*} p \leq 0.05\right)$. In addition, Nicotine-CRF intact and OVX female rats displayed more food intake relative to their respective male groups $\left({ }^{\#} p \leq 0.05\right)$. In addition, Nicotine-CRF intact females displayed significantly more food intake as compared to Nicotine-CRF OVX females ( $\left.{ }^{\dagger} p \leq 0.05\right)$. Lastly, Saline-CRF males and females displayed more food intake as compared to their respective Nicotine-CRF groups $\left({ }^{@} p \leq 0.05\right)$, an effect that was absent in OVX females.

Overexpression of CRF in the NAc altered stress-associated gene expression in a sex- and ovarian hormone-dependent manner

Figure 6 depicts changes in gene expression of CRF, CRFr1, CRFr2, and Arrb2 (Fig. 6a-d) in the NAc of male, intact female, and OVX female rats. Changes in gene expression were normalized and plotted as \% change of their respective GFP controls, such that values above $100 \%$ reflect an increase in gene expression relative to GFP controls. The analysis of CRF revealed that there was no interaction between group and virus condition $\left(F_{(2,53)}=0.18, p=\right.$ $0.84)$. However, there was a main effect of virus $\left(F_{(1,53)}=9.89, p=\right.$ 0.03 ), with all groups that received overexpression of CRF displaying higher CRF gene expression relative to respective GFP controls ( ${ }^{*} p \leq 0.05$ ). The analysis of CRFr2 revealed that there was an interaction between group and virus condition $\left(F_{(2,53)}=3.28\right.$, $p=0.05)$. Post-hoc analyses revealed that overexpression of CRF in intact females resulted in higher levels of CRFr2 as compared to GFP control $\left({ }^{*} p \leq 0.05\right)$. In both intact and OVX females, overexpression of CRF produced an increase in CRFr2 gene expression relative to CRF males ( $\left.{ }^{\#} p \leq 0.05\right)$. The analysis of CRFr1 revealed that there was no interaction between group and virus condition $\left(F_{(2,53)}=2.27, p=0.11\right)$. However, there was a main effect of virus condition $\left(F_{(1.53)}=5.99, p=0.02\right)$, which appears to be driven by the large increase in intact female relative to male $(\# p \leq 0.05)$ and OVX female $\left({ }^{\dagger} p \leq 0.05\right)$ rats that received overexpression of CRF in the NAc. The analysis of Arrb2 revealed a significant interaction between group and virus condition $\left(F_{(2,53)}=6.42, p=0.003\right)$. In both males and intact females, overexpression of CRF produced an increase in Arrb2 relative to their respective GFP controls 
Treatment Group

(A)

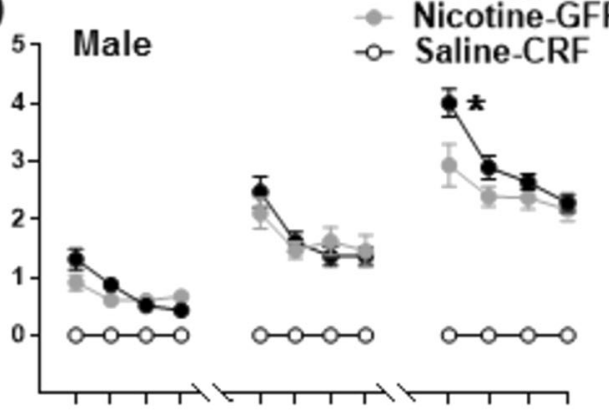

(B)

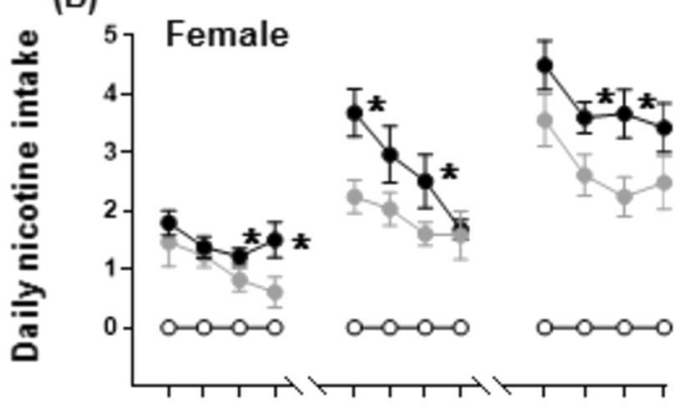

(C)

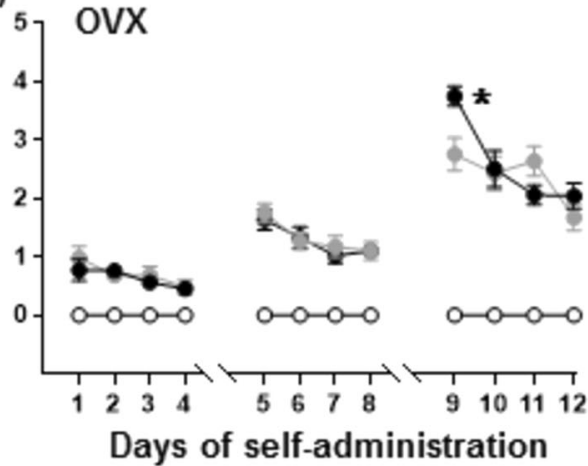

(D)

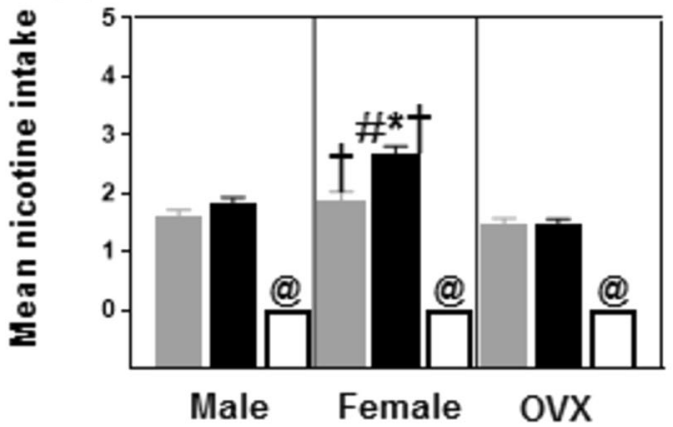

Fig. 3 Mean $( \pm S E M)$ nicotine intake across the 12 days of IVSA in male, female, and OVX female rats. During IVSA, the rats selfadministered $0.015 \mathrm{mg} / \mathrm{kg} / 0.1 \mathrm{~mL} /$ infusion on days $1-4,0.03 \mathrm{mg} / \mathrm{kg} /$ $0.1 \mathrm{~mL} /$ infusion on days $5-8$, and $0.06 \mathrm{mg} / \mathrm{kg} / 0.1 \mathrm{~mL} /$ infusion on days 9-12. The top panels (a-c) reflect daily intake and the bottom panel reflects mean intake $(\mathbf{d})$. The asterisk $\left(^{*}\right)$ denotes a significant difference between Nicotine-CRF and Nicotine-GFP groups, the number sign (\#) denotes a difference from the respective male group, the dagger ( $\dagger$ ) denotes a difference from the respective OVX female group, and the at sign (@) denotes a difference between Saline-CRF versus Nicotine-CRF rats $(p \leq 0.05)$ $\left({ }^{*} p \leq 0.05\right)$. In intact and OVX females, overexpression of CRF resulted in lower levels of Arrb2 relative to males ( ${ }^{\#} p \leq 0.05$ ). Table S1 displays delta-Ct values for all treatment groups. As a final step, the degree to which our viral manipulations overexpressed CRF in rats that received saline versus nicotine IVSA was assessed. CRF gene expression was normalized to their respective CRF groups. The analysis revealed that our viral manipulations overexpressed CRF to a similar extent across groups of rats that pressed for saline or nicotine (see Table S2).

\section{DISCUSSION}

In summary, the present study revealed that overexpression of CRF in the NAc produced greater nicotine intake across a wider range of nicotine doses in intact female versus male and OVX female rats. Regardless of virus condition, intact females displayed more inactive lever responses than males. The present study also revealed that overexpression of CRF in the NAC increased food intake relative to controls, an effect that was observed in both intact and OVX female rats. These results suggest that the effects of CRF overexpression on nicotine, but not food, intake is ovarian hormone-dependent. The magnitude of CRF gene expression was similar across groups. However, overexpression of CRF produced a larger increase in CRFr1 and CRFr2 in intact females. Also, overexpression of CRF produced a larger increase in Arrb2 in male versus intact and OVX female rats. Lastly, the immunohistochemistry results illustrated that our viral manipulation transfected neurons largely within the shell region of the NAc.

The present study revealed that intact females displayed greater nicotine IVSA as compared to males. This finding is consistent with previous reports showing sex differences in procedures involving short nicotine IVSA sessions ([27-30]; however, see [31, 32]). Our rationale for using extended-access sessions was to mimic titration patterns in human smokers and based on a recent meta-analysis showing that intact female rats display greater nicotine IVSA than males in procedures involving extended access to nicotine IVSA $[33,34]$. Thus, we suspect that sex differences in nicotine IVSA observed in the present study were facilitated by extended access and the intermittent abstinence periods that promote the development of nicotine dependence $[35,36]$. Given the role of CRF in nicotine withdrawal, the periods of abstinence likely interacted synergistically with CRF overexpression to enhance nicotine IVSA following repeated intermittent abstinence periods.

The present study also revealed that the reinforcing effects of nicotine in intact females is ovarian hormone-dependent. This is consistent with previous reports showing that OVX female rats display reductions in IVSA [37] and place preference [38] produced by nicotine as compared to intact females. Estradiol may play a role in enhancing the reinforcing effects of nicotine, given our previous finding that estradiol supplementation in OVX female rats restores nicotine IVSA to intact female levels [37]. The present study reflects a first step at examining the role of ovarian hormones in the reinforcing effects of nicotine by removing the major source of ovarian hormones in female rats. Nicotine IVSA was not compared across the 4-day estrous cycle because previous work has shown that nicotine IVSA [27] and the magnitude of CPP produced by nicotine [38] is similar across the estrous cycle. Moreover, work in our laboratory has shown that nicotine exposure and repeated vaginal lavage procedures alter the 4-day estrous cycle in female rats [39]. Ongoing work in our laboratory is assessing the contribution of ovarian hormones to the behavioral effects of nicotine via measurements of estradiol and progesterone and supplementation of these hormones in OVX female rats in a cyclical manner. This work will add important information on the therapeutic potential of these hormones in tobacco cessation approaches for women. 
(A)

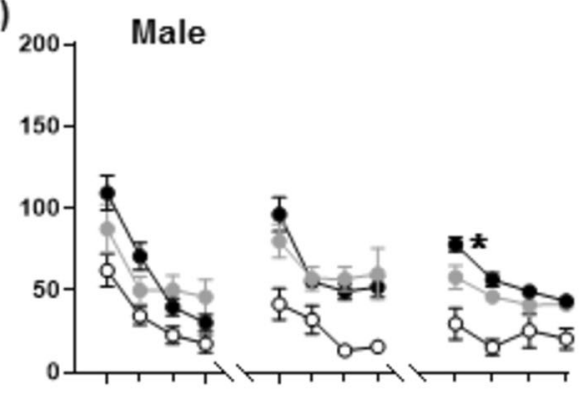

(B)

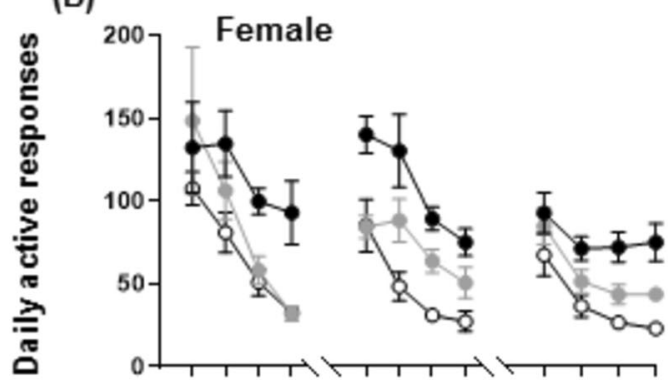

(C)

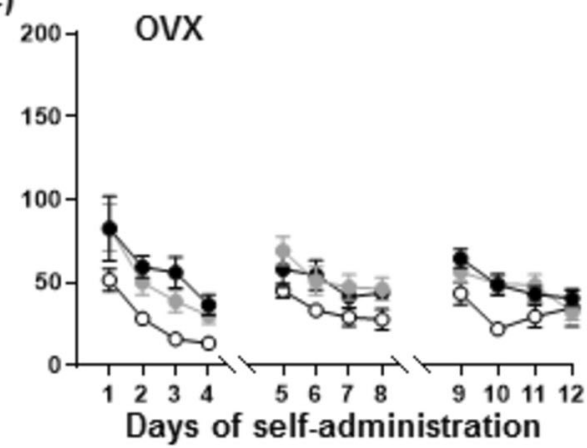

(D)

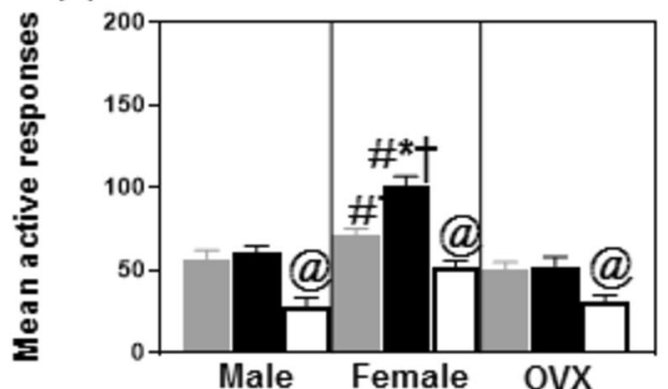

(E)

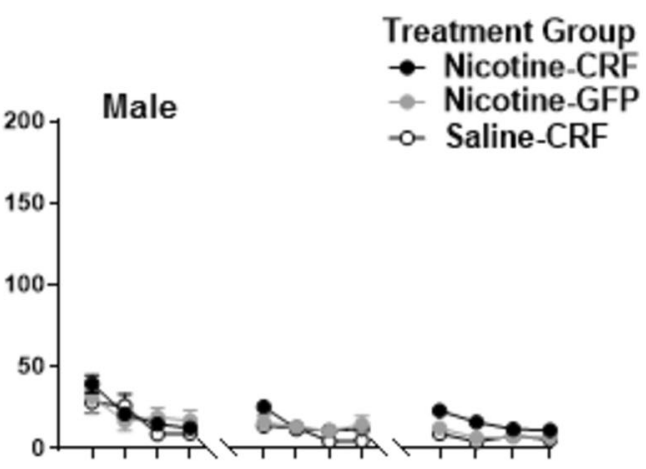

( F)

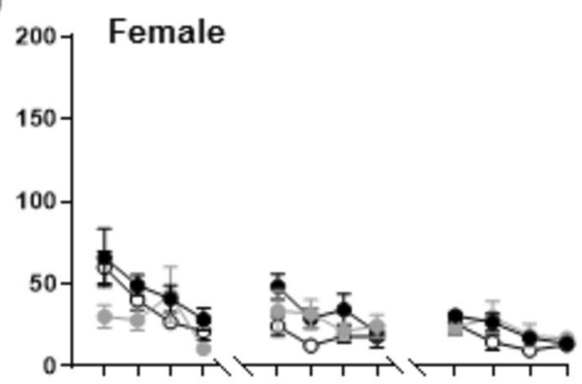

(G)

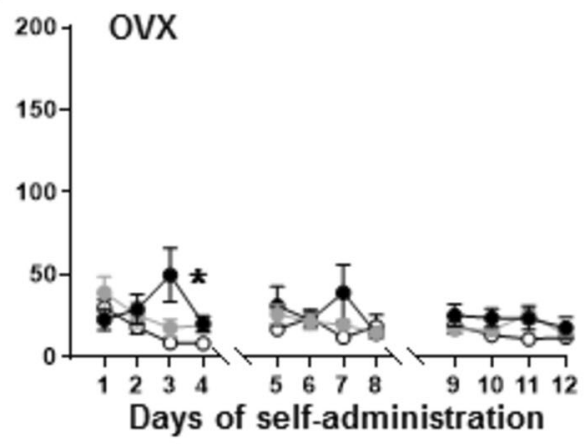

(H)

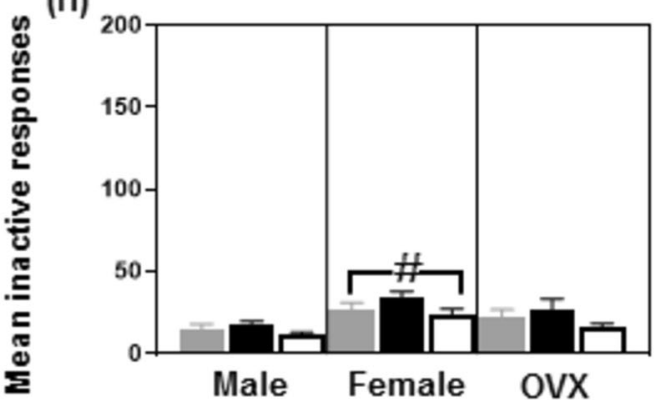

Fig. 4 Mean ( \pm SEM) active (left panels) and the inactive (right panels) responses across the 12 days of IVSA in male, female, and OVX female rats. During IVSA, the rats self-administered $0.015 \mathrm{mg} / \mathrm{kg} / 0.1 \mathrm{~mL} /$ infusion on days $1-4,0.03 \mathrm{mg} / \mathrm{kg} / 0.1 \mathrm{~mL} / \mathrm{infusion}$ on days $5-8$, and $0.06 \mathrm{mg} /$ $\mathrm{kg} / 0.1 \mathrm{~mL} /$ infusion on days 9-12. The top panels (a-c and $\mathbf{e}-\mathbf{g}$ ) reflect daily responses and the bottom panels reflect mean responses (d and h). The asterisk $(*)$ denotes a significant difference between Nicotine-CRF and Nicotine-GFP groups, the number sign (\#) denotes a difference from the respective male group, the dagger ( $\dagger$ ) denotes a difference from the respective OVX female group, and the at sign (@) denotes a difference between Saline-CRF versus Nicotine-CRF rats $(p \leq 0.05)$

The novel contribution of the present study is that overexpression of CRF in the NAc selectively increases the reinforcing effects of nicotine in intact female versus male rats. The latter effect was absent in OVX females, suggesting that the potentiating effects of CRF overexpression in the NAC are ovarian hormonedependent. A previous report revealed that microinjections of CRF into the NAc shell increased responding for a cue that signaled a sucrose reward, suggesting that CRF systems in the NAc may enhance the incentive salience of reward-related cues [40]. The present study revealed that overexpression of CRF in the NAC increased active lever responses that were signaled by a cue light to a larger extent in females versus males, an effect that may have been fueled CRF systems in the NAc that may have enhanced the salience of nicotine-associated cues in females. 
(A)

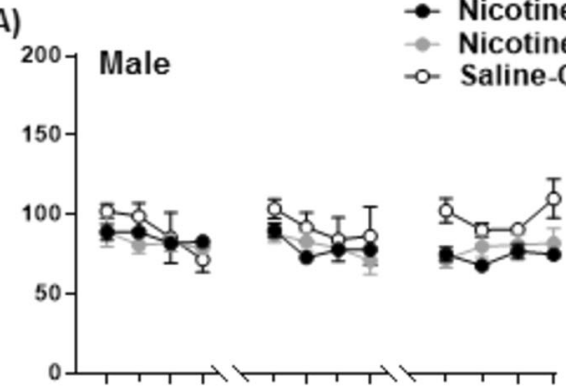

(B)

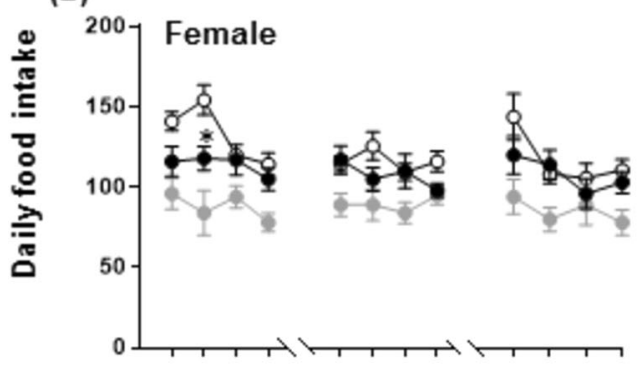

(C)

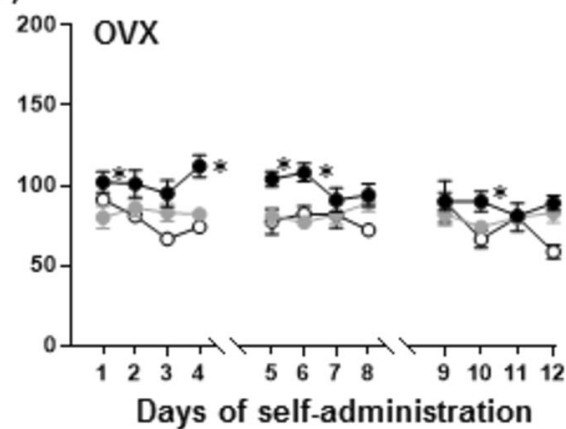

(D)

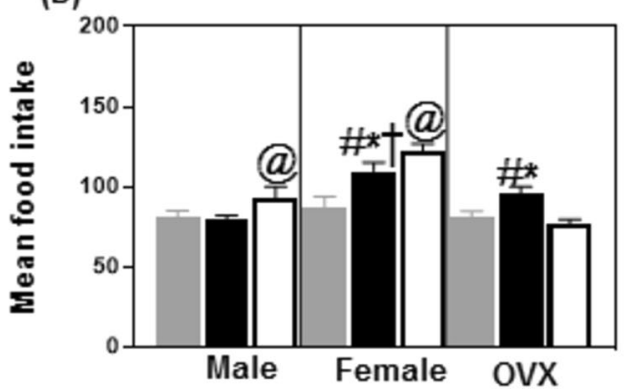

Fig. 5 Mean food intake $( \pm$ SEM) across the 12 days of IVSA in male, female, and OVX female rats. The top panels (a-c) reflect daily responses and the bottom panel reflects mean intake (d). The asterisk $\left({ }^{*}\right)$ denotes a significant difference between Nicotine-CRF and Nicotine-GFP groups, the number sign (\#) denotes a difference from the respective male group, the dagger $(\dagger)$ denotes a difference from the respective OVX female group, and the at sign (@) denotes a difference between Saline-CRF versus NicotineCRF rats $(p \leq 0.05)$

The present study extends previous work showing that CRF systems in the NAc play a key role in promoting sex differences in negative affective states produced by nicotine withdrawal. Namely, a previous study found that intact female rats display a larger upregulation of CRF gene expression than males in the NAc; however, this sex difference is not observed in the amygdala or hypothalamus [23]. Importantly, a subsequent report revealed that intact female rats display a larger upregulation of stress-associated genes in the NAc, and this effect was abolished in OVX females [41]. Together, these studies suggest that CRF systems in the NAC play a key role in promoting both the reinforcing effects of nicotine as well as the aversive states produced by nicotine withdrawal.

Previous work has established that CRF systems in the NAC modulate food-seeking behavior [42, 43]. The present study expands this literature by showing that CRF overexpression in the NAc increased food intake in female, but not male, rats. One potential reason for this sex difference is that overexpression of CRF in the NAc produced greater stress in females, and this led to higher levels of food intake as compared to males. The present study also revealed that high levels of food intake were observed in both groups of intact and OVX female rats, suggesting that the role of the NAC in modulating food reinforcement is not ovarian hormone-dependent. Previous literature suggests that stress systems influence both food and drug reinforcement [44, 45]. Indeed, a variety of stressors including yohimbine administration [46] and a variable stress procedure [47] reinstate extinguished food-seeking behavior. Previous work has also shown that the rostral portion of the NAC shell promotes feeding behavior [48], as food-port visits elicited longer-lasting changes in neural firing within the rostral, but not caudal portions of the NAC shell. Thus, the viral infusions in the present study may have preferentially activated the rostral portions of the NAc shell, resulting in increased food consumption. Previous work has shown that nicotine suppresses food intake in rodents, and the present study revealed that the increase in food intake produced by CRF overexpression was lower in male and female rats that received nicotine versus saline IVSA. Future studies might assess whether stress systems in the NAc contribute to the sex differences observed in psychological disorders involving food consumption.

The present study revealed that the magnitude of CRF overexpression in the NAc was similar across all groups. Interestingly, CRF overexpression enhanced gene expression of CRFr1 and CRFr2 to a greater extent in intact female versus male rats. The larger increase in CRF receptors in intact females may have occurred via two distinct mechanisms. First, males displayed greater levels of Arrb2 relative to both groups of females, which is consistent with previous literature [49]. Thus, females may display more stress receptors in response to reduced internalization of these sites. Consistent with this hypothesis, female rats display reduced expression of Arrb2 than males [50], which internalizes CRFr1 [51] and CRFr2 [52] receptors. Previous reports have found that CRFr1 receptors are more often coupled to GTP binding sites in females as compared to males that display greater coupling to Arrb2 sites [49, 53]. Thus, females might display enhanced CRF system modulation of the NAc due to an increase in CRF receptor availability and activation. Second, the presence of estrogen enhances the expression of CRFr1 and CRFr2 expression in breast cancer cells [54]. Thus, estrogen in intact females may enhance the expression of CRF receptors in the NAc.

The present study revealed that the reinforcing effects of nicotine were greater in females that received overexpression of CRF in the NAc. We propose an estradiol (E2)-based mechanism in the NAc based on work described in $[55,56]$. Specifically, estrogen receptor beta $(E R \beta)$ is located on the terminals of medium spiny striatal neurons that have recurrent collaterals on dopamine terminals [57]. It is suggested that E2 inhibits the activity of inhibitory medium spiny neurons in the NAc that are primarily of gamma-aminobutyric acid (GABA) origin. In the presence of E2, striatal GABA release is decreased, which removes GABAergic inhibition and increases dopamine release in the NAC $[55,58]$. Additionally, there is a dense population of CRFr1 receptors that are colocalized with tyrosine hydroxylase-immunoreactive fibers, 


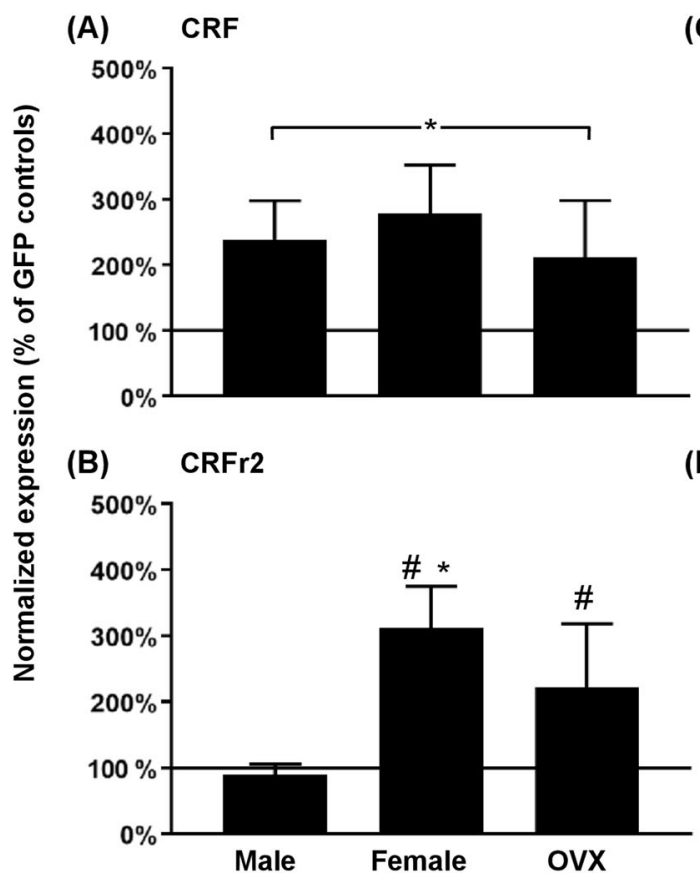

(C) CRFr1

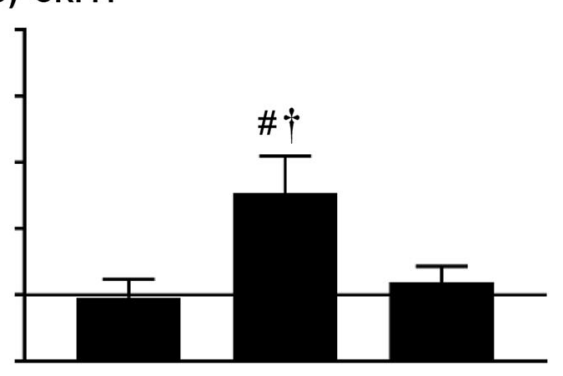

(D) Arrb2

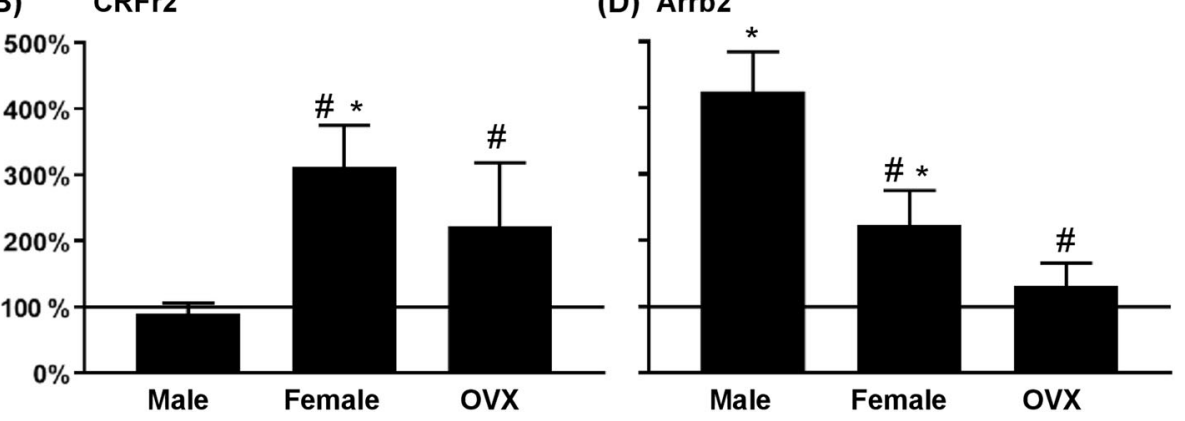

Fig. 6 Gene expression ( \pm SEM) of CRF (a), CRFr2 (b), CRFr1 (c), and Arrb2 (d) in the NAc of male, female, and OVX female rats. The data were normalized and then expressed as a percentage of their respective GFP control levels. The asterisk $\left(^{*}\right)$ denotes a significant difference between Nicotine-CRF and Nicotine-GFP groups, the number sign (\#) denote a difference from males, and the dagger ( $($ ) denotes a difference from OVX females $(p \leq 0.05)$

indicating the presence of CRFr1 receptors on dopamine terminals in the NAc [17]. It is suggested that co-activation of ER $\beta$ and CRFr1 receptors in females provides major inhibition of GABAergic activity, which would result in greater dopamine release in the NAc and stronger reinforcing effects of nicotine in females. Indeed, female rats display a larger increase in NAc dopamine following nicotine administration as compared to males [59].

This study reflects an important first step towards understanding the neural circuits by which stress promotes tobacco use in females. Specifically, we showed that the NAc is a sexually dimorphic structure where CRF and ovarian-hormone systems synergistically amplify the reinforcing effects of nicotine in females. While the present study suggests that the NAc is an important locus of regulation for the behavioral effects of nicotine, it is unlikely that this region works in isolation. Indeed, gonadal hormones have been shown to modulate several regions in the limbic system, including the BNST [60] and CeA [19] that modulate the behavioral effects of nicotine. Another brain region to consider is the medial preoptic area (mPOA), which is a primary locus for the integration of endocrine stimulation. Indeed, intra-mPOA E2 administration enhances cocaine-induced increases in NAc dopamine levels [61]. Future studies are needed to understand the mechanisms by which various neuro-endocrine factors modulate sex differences and reward processing beyond the NAc. This work will ultimately lead to a better understanding of the mechanisms by which stress and ovarian-hormone systems promote nicotine use in females. This preclinical research is important, as women are more likely to suffer from stressassociated disorders and use tobacco to cope with negative affective states [2-5]. Our work suggests that the most effective treatment approaches for smoking cessation in women should reduce stress, possibly via pharmacological interventions that reduce CRF systems. Any potential differences in mechanisms of susceptibility could provide novel targets for more effective tobacco cessation strategies that will reduce health disparities in women.

\section{FUNDING AND DISCLOSURE}

Funding for this project was provided by National Institutes of Health grants (R01-DA021274 and R25-DA033613) to LEO. This project was also supported by a Ruth L. Kirschstein pre-doctoral fellowship (F31-DA046126) to KPU and a post-doctoral training contract (HHSN271201600057C) to LEO that supported VLC. Work in the Biological Sciences department was supported by $\mathrm{NIH}$ grants SC1-GM127251 and R01-MH114961. Confocal imaging was performed in the Cytometry, Screening, and Imaging Core of the Border Biomedical Research Center, which is supported by the National Institute on Minority Health and Health Disparities (5G12MD007592). The authors declare no competing interests.

\section{ADDITIONAL INFORMATION}

Supplementary Information accompanies this paper at (https://doi.org/10.1038/ s41386-019-0543-0).

Publisher's note Springer Nature remains neutral with regard to jurisdictional claims in published maps and institutional affiliations.

\section{REFERENCES}

1. Samet JM. Tobacco smoking: the leading cause of preventable disease worldwide. Thorac Surg Clin. 2013;23:103-12.

2. Perkins KA, Donny E, Caggiula AR. Sex differences in nicotine effects and selfadministration: review of human and animal evidence. Nicotine Tob Res. 1999;1:301-15.

3. Perkins KA, Giedgowd GE, Karelitz JL, Conklin CA, Lerman C. Smoking in response to negative mood in men versus women as a function of distress tolerance. Nicotine Tob Res. 2012;14:1418-25.

4. Perkins KA, Karelitz JL, Giedgowd GE, Conklin CA. Negative mood effects on craving to smoke in women versus men. Addict Behav. 2013;38:1527-31.

5. Mykletun A, Overland S, Aaro LE, Liabo HM, Stewart R. Smoking in relation to anxiety and depression: evidence from a large population survey: the HUNT study. Eur Psychiatry. 2008;23:77-84.

6. Brook JS, Zhang C, Brook DW, Koppel J, Whiteman M. Psychosocial predictors of nicotine dependence among women during their mid-sixties. Am J Addict. 2012;21:302-12 
7. Dedert EA, Calhoun PS, Harper LA, Dutton CE, McClernon FJ, Beckham JC. Smoking withdrawal in smokers with and without posttraumatic stress disorder. Nicotine Tob Res. 2012;14:372-6.

8. Langdon KJ, Leventhal AM. Posttraumatic stress symptoms and tobacco abstinence effects in a non-clinical sample: evaluating the mediating role of negative affect reduction smoking expectancies. J Psychopharmacol. 2014;28:1009-17.

9. Childs $\mathrm{E}$, de Wit H. Hormonal, cardiovascular, and subjective responses to acute stress in smokers. Psychopharmacol (Berl). 2009;203:1-12.

10. Feltenstein MW, Ghee SM, See RE. Nicotine self-administration and reinstatement of nicotine-seeking in male and female rats. Drug Alcohol Depend. 2012;121: 240-6.

11. Buczek Y, Le AD, Wang A, Stewart J, Shaham Y. Stress reinstates nicotine seeking but not sucrose solution seeking in rats. Psychopharmacol (Berl). 1999;144:183-8.

12. Leao RM, Cruz FC, Planeta CS. Exposure to acute restraint stress reinstates nicotine-induced place preference in rats. Behav Pharmacol. 2009;20:109-13.

13. Zislis G, Desai TV, Prado M, Shah HP, Bruijnzeel AW. Effects of the CRF receptor antagonist D-Phe CRF(12-41) and the alpha2-adrenergic receptor agonist clonidine on stress-induced reinstatement of nicotine-seeking behavior in rats. Neuropharmacology. 2007;53:958-66.

14. Bruijnzeel AW, Prado M, Isaac S. Corticotropin-releasing factor-1 receptor activation mediates nicotine withdrawal-induced deficit in brain reward function and stress-induced relapse. Biol Psychiatry. 2009;66:110-7.

15. Swanson LW, Sawchenko PE, Rivier J, Vale WW. Organization of ovine corticotropin-releasing factor immunoreactive cells and fibers in the rat brain: an immunohistochemical study. Neuroendocrinology. 1983;36:165-86.

16. Albrechet-Souza L, Hwa LS, Han X, Zhang EY, DeBold JF, Miczek KA. Corticotropin releasing factor binding protein and CRF2 receptors in the ventral tegmental area: modulation of ethanol binge drinking in C57BL/6J mice. Alcohol Clin Exp Res. 2015;39:1609-18.

17. Lemos JC, Wanat MJ, Smith JS, Reyes BA, Hollon NG, Van Bockstaele EJ, et al. Severe stress switches CRF action in the nucleus accumbens from appetitive to aversive. Nature. 2012;490:402-6.

18. Paxinos G, Watson C. The Rat Brain in Stereotaxic Coordinates. 7th ed. Cambridge, MA: Academic Press; 2013.

19. Qi X, Shan Z, Ji Y, Guerra V, Alexander JC, Ormerod BK, et al. Sustained AAVmediated overexpression of CRF in the central amygdala diminishes the depressive-like state associated with nicotine withdrawal. Transl Psychiatry. 2014;4:e385

20. Natividad LA, Torres OV, Friedman TC, O'Dell LE. Adolescence is a period of development characterized by short- and long-term vulnerability to the rewarding effects of nicotine and reduced sensitivity to the anorectic effects of this drug. Behav Brain Res. 2013;257:275-85.

21. Chen SA, O'Dell LE, Hoefer ME, Greenwell TN, Zorrilla EP, Koob GF. Unlimited access to heroin self-administration: independent motivational markers of opiate dependence. Neuropsychopharmacology. 2006;31:2692-707.

22. Sidhu KS. Basis for body weight exponent $(0.75)$ as a scaling factor in energy metabolism and risk assessment. J Appl Toxicol. 1992;12:309-10.

23. Torres OV, Gentil LG, Natividad LA, Carcoba LM, O'Dell LE. Behavioral, biochemical, and molecular indices of stress are enhanced in female versus male rats experiencing nicotine withdrawal. Front Psychiatry. 2013;4:38.

24. Khan AM, Walker EM, Dominguez N, Watts AG. Neural input is critical for arcuate hypothalamic neurons to mount intracellular signaling responses to glycemic challenges in male rats: implications for communication within feeding and metabolic control networks. Endocrinology. 2014;155:405-16.

25. Livak KJ, Schmittgen TD. Analysis of relative gene expression data using realtime quantitative PCR and the 2(-Delta Delta C(T)) Method. Methods. 2001;25: 402-8.

26. Sierra-Fonseca JA, Najera O, Martinez-Jurado J, Walker EM, Varela-Ramirez A, Khan AM, et al. Nerve growth factor induces neurite outgrowth of PC12 cells by promoting Gbetagamma-microtubule interaction. BMC Neurosci. 2014;15:132.

27. Donny EC, Caggiula AR, Mielke MM, Booth S, Gharib MA, Hoffman A, et al. Nicotine self-administration in rats on a progressive ratio schedule of reinforcement. Psychopharmacol (Berl). 1999;147:135-42.

28. Chaudhri N, Caggiula AR, Donny EC, Booth S, Gharib MA, Craven LA, et al. Sex differences in the contribution of nicotine and nonpharmacological stimuli to nicotine self-administration in rats. Psychopharmacol (Berl). 2005; 180:258-66

29. Rezvani AH, Eddins D, Slade S, Hampton DS, Christopher NC, Petro A, et al. Neonatal 6-hydroxydopamine lesions of the frontal cortex in rats: persisting effects on locomotor activity, learning and nicotine self-administration. Neuroscience. 2008;154:885-97.

30. Sanchez V, Moore CF, Brunzell DH, Lynch WJ. Sex differences in the effect of wheel running on subsequent nicotine-seeking in a rat adolescent-onset selfadministration model. Psychopharmacol (Berl). 2014;231:1753-62.
31. Pittenger ST, Swalve N, Chou S, Smith MD, Hoonakker AJ, Pudiak CM, et al. Sex differences in neurotensin and substance $P$ following nicotine self-administration in rats. Synapse. 2016;70:336-46.

32. Swalve N, Smethells JR, Carroll ME. Sex differences in the acquisition and maintenance of cocaine and nicotine self-administration in rats. Psychopharmacol (Berl). 2016;233:1005-13.

33. O'Dell LE, Chen SA, Smith RT, Specio SE, Balster RL, Paterson NE, et al. Extended access to nicotine self-administration leads to dependence: Circadian measures, withdrawal measures, and extinction behavior in rats. J Pharm Exp Ther. 2007; 320:180-93.

34. Flores RJ, Uribe KP, Swalve N, O'Dell LE. Sex differences in nicotine intravenous self-administration: a meta-analytic review. Physiol Behav. 2019;203:42-50.

35. O'Dell LE, Koob GF. 'Nicotine deprivation effect' in rats with intermittent 23-hour access to intravenous nicotine self-administration. Pharm Biochem Behav. 2007;86:346-53.

36. Cohen A, Treweek J, Edwards S, Leao RM, Schulteis G, Koob GF, et al. Extended access to nicotine leads to a CRF1 receptor dependent increase in anxiety-like behavior and hyperalgesia in rats. Addict Biol. 2015;20:56-68.

37. Flores RJ, Pipkin JA, Uribe KP, Perez A, O'Dell LE. Estradiol promotes the rewarding effects of nicotine in female rats. Behav Brain Res. 2016;307:258-63.

38. Torres OV, Natividad LA, Tejeda HA, Van Weelden SA, O'Dell LE. Female rats display dose-dependent differences to the rewarding and aversive effects of nicotine in an age-, hormone-, and sex-dependent manner. Psychopharmacol (Berl). 2009;206:303-12.

39. Flores RJ, Cruz B, Uribe KP, Carcoba LM, O'Dell LE. Sex differences and the role of ovarian hormones in modulating the behavioral effects of nicotine in rodent models. In: Becker JB, Tollkuhn J, editors. Sex differences in the brain: balancing sex in preclinical research. Washington, DC: Society of Neuroscience; 2018. p. 59-67.

40. Pecina S, Schulkin J, Berridge KC. Nucleus accumbens corticotropin-releasing factor increases cue-triggered motivation for sucrose reward: paradoxical positive incentive effects in stress? BMC Biol. 2006;4:8.

41. Torres OV, Pipkin JA, Ferree $P$, Carcoba LM, O'Dell LE. Nicotine withdrawal increases stress-associated genes in the nucleus accumbens of female rats in a hormone-dependent manner. Nicotine Tob Res. 2015;17:422-30.

42. Salamone JD, Correa M, Farrar A, Mingote SM. Effort-related functions of nucleus accumbens dopamine and associated forebrain circuits. Psychopharmacol (Berl). 2007;191:461-82.

43. Nicola SM. Reassessing wanting and liking in the study of mesolimbic influence on food intake. Am J Physiol Regul Integr Comp Physiol. 2016;311:R811-40.

44. Volkow ND, O'Brien CP. Issues for DSM-V: should obesity be included as a brain disorder? Am J Psychiatry. 2007;164:708-10.

45. Volkow ND, Wang GJ, Tomasi D, Baler RD. Obesity and addiction: neurobiological overlaps. Obes Rev. 2013;14:2-18.

46. Nair SG, Gray SM, Ghitza UE. Role of food type in yohimbine- and pellet-priminginduced reinstatement of food seeking. Physiol Behav. 2006:88:559-66.

47. Schepers ST, Bouton ME. Stress as a context: stress causes relapse of inhibited food seeking if it has been associated with prior food seeking. Appetite. 2019; 132:131-8.

48. Reed SJ, Lafferty CK, Mendoza JA, Yang AK, Davidson TJ, Grosenick L, et al. Coordinated reductions in excitatory input to the nucleus accumbens underlie food consumption. Neuron. 2018;99:1260-73 e1264.

49. Bangasser DA, Curtis A, Reyes BA, Bethea $\Pi$, Parastatidis I, Ischiropoulos $\mathrm{H}$, et al. Sex differences in corticotropin-releasing factor receptor signaling and trafficking: potential role in female vulnerability to stress-related psychopathology. Mol Psychiatry. 2010;15:896-904.

50. Valentino RJ, Bangasser D, Van Bockstaele EJ. Sex-biased stress signaling: the corticotropin-releasing factor receptor as a model. Mol Pharmacol. 2013;83: 737-45.

51. Valentino RJ, Van Bockstaele E, Bangasser D. Sex-specific cell signaling: the corticotropin-releasing factor receptor model. Trends Pharm Sci. 2013;34:437-44.

52. Hauger RL, Risbrough V, Oakley RH, Olivares-Reyes JA, Dautzenberg FM. Role of CRF receptor signaling in stress vulnerability, anxiety, and depression. Ann N Y Acad Sci. 2009;1179:120-43.

53. Bangasser DA, Dong H, Carroll J, Plona Z, Ding H, Rodriguez L, et al. Corticotropinreleasing factor overexpression gives rise to sex differences in Alzheimer's disease-related signaling. Mol Psychiatry. 2017;22:1126-33.

54. Lal S, Allan A, Markovic D, Walker R, Macartney J, Europe-Finner N, et al. Estrogen alters the splicing of type 1 corticotropin-releasing hormone receptor in breast cancer cells. Sci Signal. 2013;6:ra53.

55. Becker JB, Hu M. Sex differences in drug abuse. Front Neuroendocrinol. 2008;29:36-47

56. Becker JB, Perry AN, Westenbroek C. Sex differences in the neural mechanisms mediating addiction: a new synthesis and hypothesis. Biol Sex Differ. 2012;3:14. 
Overexpression of corticotropin-releasing factor in the nucleus accumbens... $\mathrm{KP}$ Uribe et al.

57. Mermelstein PG, Becker JB, Surmeier DJ. Estradiol reduces calcium currents in rat neostriatal neurons via a membrane receptor. J Neurosci. 1996;16:595-604.

58. Hu M, Watson CJ, Kennedy RT, Becker JB. Estradiol attenuates the K+-induced increase in extracellular GABA in rat striatum. Synapse. 2006;59:122-4.

59. Pogun S. Sex differences in brain and behavior: emphasis on nicotine, nitric oxide and place learning. Int J Psychophysiol. 2001;42:195-208.
60. Qi X, Guzhva L, Yang Z, Febo M, Shan Z, Wang KKW, et al. Overexpression of CRF in the BNST diminishes dysphoria but not anxiety-like behavior in nicotine withdrawing rats. Eur Neuropsychopharmacol. 2016;26:1378-89.

61. Tobiansky DJ, Will RG, Lominac KD, Turner JM, Hattori T, Krishnan K, et al. Estradiol in the preoptic area regulates the dopaminergic response to cocaine in the nucleus accumbens. Neuropsychopharmacol. 2016;41:1897-906. 